\title{
ON THE ARITHMETIC AND HARMONIC AVERAGES IN NONDISCRETE SETTING
}

\author{
FERENC MóRICZ
}

Abstract. In recent years, the almost sure central limit theorem attracted widespread attention in Probability Theory. It involves the harmonic (also called logarithmic) averages of a numerical sequence. In our previous paper [2], we investigated the convergence behavior of the sequence of harmonic averages of a given numerical sequence from the viewpoint of Summability Theory. In the present paper, our aim is to extend this investigation from the discrete to nondiscrete setting. Given a real or complex-valued function which is locally integrable on $\mathbb{R}_{+}$, we give necessary and sufficient conditions for the existence of the finite limit of its arithmetic or harmonic averages, respectively. These characterizations may be useful in the investigation of the almost sure behavior of Stochastic Processes.

Mathematics subject classification (2010): Primary 40A10, 40C10; Secondary 26D15, 60F05, $60 \mathrm{H} 99$. Keywords and phrases: arithmetic averages; locally integrable functions; higher order harmonic averages; inclusion theorems.

\section{REFERENCES}

[1] I. BERKes. E. CSÁKI AND L. HoRVÁth, An almost sure central limit theorem under minimal conditions, Stat. Probab. Letters, 37 (1998), 67-76.

[2] F. MórICZ, On the harmonic averages of numerical sequences, Arch. Math. (Basel), 86 (2006), 375384.

[3] P. RÉvÉSZ, The Laws of Large Numbers, Akadémiai Kiadó, Budapest, 1967.

[4] A. Zygmund, Trigonometric Series, Vol. I, Cambridge Univ. Press, 1959. 